\title{
Association between genotype and phenotype of virulence gene in Van der Woude syndrome families
}

\author{
SHOUXIA LI ${ }^{1 *}$, XUEQIANG ZHANG $^{1 *}$, DINGLI CHEN ${ }^{1}$, WEI ZHAO ${ }^{2}$, XIAOFANG ZHANG $^{1}$, \\ JIANJUN JIAO ${ }^{1}$, LILI GUO ${ }^{1}$, LEI YIN ${ }^{1}$, XUEDONG SONG ${ }^{1}$, CHUN LIANG ${ }^{1}$ and CAIXIA SUN ${ }^{1}$ \\ ${ }^{1}$ Department of Clinical Laboratory, Handan Central Hospital, Handan, Hebei 056001; ${ }^{2}$ First School of \\ Clinical Medicine, Southern Medical University, Guangzhou, Guangdong 510515, P.R. China
}

Received May 17, 2016; Accepted April 4, 2017

DOI: $10.3892 / \mathrm{mmr} .2017 .7978$

\begin{abstract}
Members from two Van der Woude syndrome (VWS) families were screened to determine the prevalence of interferon regulatory factor 6 (IRF6) as a disease-causing gene and to analyze the interrelationships between patient genotype and phenotype. The peripheral blood of 24 members from two VWS families and 200 control samples were collected. The family members were interviewed for medical histories and other clinical abnormalities using questionnaires. Polymerase chain reaction was directly performed on the peripheral blood to screen for the coding region of the IRF6 gene. Of the 24 family members, a total of 6 patients had mutations of IRF6 gene. c.1234C >T (p.R412X) heterozygous mutation was detected in 3 members of family 1 . In families 2 and 3, members carried the c.1210G $>$ A (p.E404K) heterozygous mutations. The other members of the families, were wild type (wt/wt) for IRF6. Genetic testing demonstrated that the disease mutations c. $1234 \mathrm{C}>\mathrm{T}$ and $\mathrm{c} .1210 \mathrm{G}>\mathrm{A}$ co-segregated with the two families' pathogenic mutations. The existence of genetic heterogeneity and the complexity of the clinical phenotype was demonstrated in Chinese VWS patients.
\end{abstract}

\section{Introduction}

Van der wood Syndrome [VWS; Online Mendelian Inheritance in Man (www.omim.org) 119300] is an autosomal dominant inheritance of cleft lip and palate deformities. The primary clinical features of VWS are lower lip fistula, teeth agenesis or pits, simple cleft palate, simple cleft lip or cleft palate and cleft palate merger, and several of the features may appear at

Correspondence to: Professor Shouxia Li, Department of Clinical Laboratory, Handan Central Hospital, 59 CongTai North Road, CongTai, Handan, Hebei 056001, P.R. China

E-mail: sxdoccn@126.com

${ }^{*}$ Contributed equally

Key words: interferon regulatory factor 6, Van der Woude syndrome, cleft lip with or without cleft palate, gene sequencing, mutation the same time. VWS is a syndrome in cleft lip and palate, and accounts for $2 \%$ of all patients with cleft lip and palate (1). Kondo et al (2) succeeded in cloning the interferon regulatory factor (IRF) 6 gene that was the disease-causing gene of VWS, and VWS has been considered to be the best genetic study model of non-syndromic cleft lip with or without cleft palate. In 1986, the global incidence of VWS was $1 \sim 3 / 30,000$, and the incidence in newborns was 1/100,000-1/40,000 (3), but there was a big difference in phenotypes $(3,4)$. The clinical phenotype of VWS patients differs with families and even among VWS patients within the same family. Due to the different phenotypes, the clinical diagnosis of VWS has often been confused with non-syndromic cleft lip with or without cleft palate (NSCL/P), with the lower lip pits easily mistaken for bite marks. Peyrard-Janvid et al (5) studied 17 families from Sweden, Finland, Norway, Thailand and Singapore, and reported that $80 \%$ of VWS patients exhibited an IRF6 gene mutation, thus demonstrating that it was a reliable marker of VWS. IRF6 in VWS has not been so well reported in China, although previous studies have confirmed that IRF6 is associated with VWS in a clinical study of a VWS family $(6,7)$. By a joint analysis of clinical data and genetic testing results of a VWS family, the present study aimed to clarify the nosogenesis of these patients from the perspective of genetics, and analyze the association between genotype and phenotype. It also aimed to clarify the VWS pathogenic mutations of Chinese VWS patients, and to identify the suspected gene mutation areas of interest. The pathogenic gene detection of VWS may be used for aiding clinical assessment in the future, and for prenatal diagnosis.

\section{Materials and methods}

General. The probands from two families were inpatients in the oral and maxillofacial surgery ward of Handan Central Hospital (Handan, China). The two families had 24 members, including 6 with VWS. At the same time, 200 peripheral blood samples from normal individuals who did not possess a family history of cleft lip or palate or other immune organic diseases were collected between January and March 2014; these individuals were recruited from the Department of Clinical Laboratory, Handan Central Hospital (Hebei, China). The genetic and patient information of probands were provided by their parents. The present study was conducted in accordance 
with the declaration of Helsinki and with approval from the Ethics Committee of Handan Central Hospital. Written informed consent was obtained from all participants' guardians and the 200 normal individuals.

Family 1 (VWS1): Proband A (III:3; Figs. 1A and 2), male, 2 years old, had congenital cleft palate and bilateral labium fistula. His mother did not experience a cold or a fever or use drugs during pregnancy. There were 3 generations and 11 members in this family. The VWS patients in this family were 3 in total, and distributed in each generation. Pedigree analysis was compatible with the law of autosomal dominant inheritance. Through detailed examination by maxillofacial surgeons, congenital malformations, and cardiovascular, cerebrovascular and mental diseases were excluded for the patients in this family. Although the patients in this family expressed typical symptoms of VWS, the individual phenotype in each generation was different. The symptoms of the patient in the first generation (I:2) were characterized by simple red small pits on the lower lip, not easy to distinguish from bite marks. The patients in the second (II:3) and third (III:3) generations demonstrated serious cleft lip and palate deformities and lower lip fistula.

Family 2 (VWS2): Proband B (III:7; Figs. 1B and 3), male, 3 years old, had congenital cleft soft palate. His mother did not experience a cold or a fever or use drugs during pregnancy. There were 3 generations and 13 members in this family and congenital malformations, and cardiovascular, cerebrovascular and mental diseases were excluded. The VWS patients in this family were 3 in total, and appeared in the second and third generation. Pedigree analysis was also compatible with law of autosomal dominant inheritance. Through detailed examination by maxillofacial surgeons, the symptoms of VWS patients in the second generation were characterized by bite marks in the red defects of the lower lip and occult cleft palate. The VWS patients in the third generation were siblings. The elder sister (III:3) demonstrated cleft palate and lower lip fistula. The young brother was the proband and demonstrated cleft soft palate.

Medical history collection. The genetic and patient information of probands were filled in registration forms by their parents. The information in the hereditary information registration form included general information concerning the patients, pregnancy risk factors, pregnancy situation, family history, the VWS patient situation and laboratory tests. The information in the patient information forms included the patients' general information, general situation, history of mother's pregnancy (including history of infection, drug administration and childbirth), physical examination (including systemic physical examination and professional oral medical examination) and various clinical data. All family members from whom peripheral blood samples were collected provided written informed consent. The family members who agreed to donate blood were provided with a medical examination and $5 \mathrm{ml}$ of peripheral venous blood was extracted for subsequent testing.

Genomic DNA extraction. The genomic DNA was extracted by AxyPrep blood genomic DNA extraction kit (Axygen Scientific, Inc., Union City, CA, USA) in strict accordance with the manufacturer's protocol. DNA concentration and purity were detected by NanoDrop 1000 determination
(Thermo Fisher Scientific, Inc., Wilmington, DE, USA). When the extraction process was complete, all DNA samples were diluted to $10 \mathrm{ng} / \mu \mathrm{l}$ in 96 well plates and stored at $-20^{\circ} \mathrm{C}$.

Gene sequencing. The amplification primer sequences of IRF6 were designed by online software Primer 3 version 3.0.0 (www.biotools.umassmed.edu/bioapps/primer3_www .cgi). The amplified fragment was the exons between 3 and 9 , since exons 1 and 2 were encoding fragments. Polymerase chain reaction (PCR) primer sequences and amplified fragment length are listed in Table I. Primers were synthesized by Invitrogen (Thermo Fisher Scientific, Inc., Waltham, MA, USA). The PCR reaction conditions were: Pre-denaturation at $94^{\circ} \mathrm{C}$ for $5 \mathrm{~min}$, denaturation at $94^{\circ} \mathrm{C}$ for $30 \mathrm{sec}$, annealing at $6^{\circ} \mathrm{C}$ for $30 \mathrm{sec}$, extension at $72^{\circ} \mathrm{C}$ for $35 \mathrm{sec}, 35$ cycles in total; extension at $72^{\circ} \mathrm{C}$ for $5 \mathrm{~min}$. The PCR product was subjected to electrophoresis on $1.5 \%$ agarose gel and the results were analyzed by a gel imaging and analysis system (Gel Doc ${ }^{\mathrm{TM}} \mathrm{EZ}$; Bio-Rad Laboratories, Inc., Hercules, CA, USA). PCR products were sequenced by an ABI 3730 (Applied Biosystems; Thermo Fisher Scientific, Inc.), and the mutation positive samples were sequence reversed for verification. The sequencing results were compared with the standard IRF6 genetic sequence on National Center for Biotechnology Information (https://www .ncbi.nlm.nih.gov; NM_006147) by Mutation Surveyor software version 4.0 (SoftGenetics, LLC., State College, PA, USA).

\section{Results}

Clinical examination results. There were 6 VWS patients in the two families that comprised 24 individuals in total. Of the 6 patients, 5 (83.3\%) presented cleft lip and palate, 5 (83.3\%) presented labial fistula and 1 (III:7) in the second family was without labial fistula. These VWS patients presented no teeth dysplasia or other organ deformity, although the phenotypic lip fistula with cleft lip was common. Although the patients belonged to the same family, their clinical phenotype differed: 3 patients in the first family all had lower lip fistula or labium fistula with cleft lip and palate, patient I:2 was the only one exhibiting bilateral labium fistula, patient II:3 demonstrated degree II right cleft palate and patient III:3 demonstrated degree III left cleft palate. Two of the total 3 patients in the second family had lower lip bite marks. Patient II:3 presented lower lip bite marks on the sides and cracked palate, patient III:3 was characterized by degree II left cleft palate for and lower lip bite marks, and patient III:7 demonstrated soft cleft palate alone.

Genetic testing results. The gene sequencing of IFR6 between exons 3 and 9 was conducted on 14 members of the 2 families. The results demonstrated that the 6 VWS patients all had genetic mutations. The 3 patients in the first family all carried c.1234C $>\mathrm{T}$ (p.R412X) heterozygous mutation on exon 9 of the IRF6 gene (Fig. 4). In family 2, 3 patients all carried c.1210G $>$ A (p.E404K) heterozygous mutation on exon 9 of the IRF6 gene (Fig. 5). In addition, II:3 and III:3 in family 2 carried the rs2235373 single nucleotide polymorphism (SNP) on exon 7 of the IRF6 gene. Other family members who were not VWS patients did not carry IRR6 gene mutations. Genetic testing results demonstrated that the mutation and disease exhibited co-segregation. 


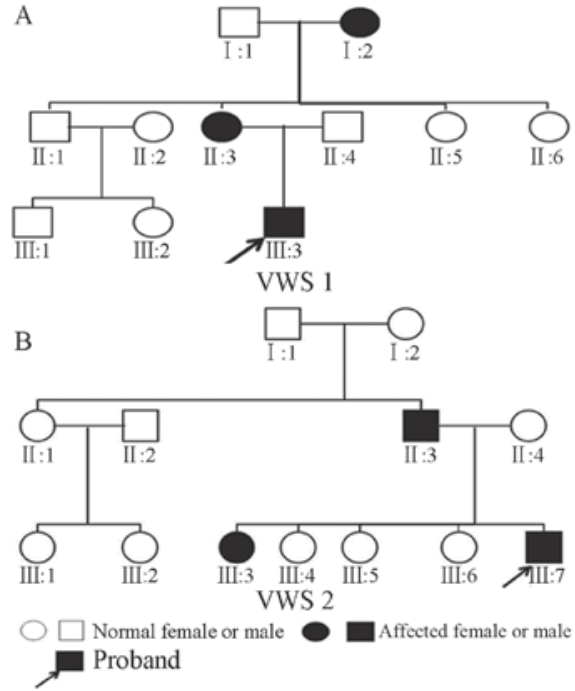

Figure 1. Genogram of the two families. (A) VWS family 1 and (B) VWS family 2 . Van der Woude syndrome.

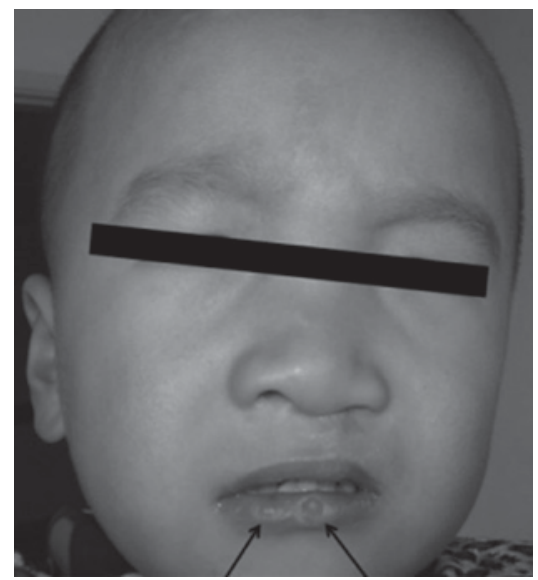

Figure 2. Proband III:3 of the Van der Woude syndrome 1 family. Arrows indicate lower lip fistula.

Normal test results. In order to rule out that the two mutation sites detected in the two families were not SNPs in local people, the exon 9 gene sequencing of IFR6 was conducted on 200 local normal individuals and the c.1234C $>\mathrm{T}$ and c.1210G $>$ A mutations were not observed.

\section{Discussion}

VWS, an autosomal dominant inherited clefting syndrome first described by Demarquay in 1845 , is a rare congenital syndrome (8). The characteristics of the phenotype are labium fistula with cleft lip and palate. In VWS, the formation of congenital lip pits is associated with the occurrence of labium fistula with cleft lip and palate; this is a common clinical problem that occurs in $80 \%$ of patients with VWS (9). Therefore, certain patients with VWS were characterized only by simple cleft lip or cleft palate, and this made VWS and NSCL/P indistinguishable from the outward appearance of patients. However, for the congenital anomaly of epidemiology and etiology, accurate phenotyping is important. If the heterogeneous group is regarded as a single group, the effect of detection may be weakened (10).

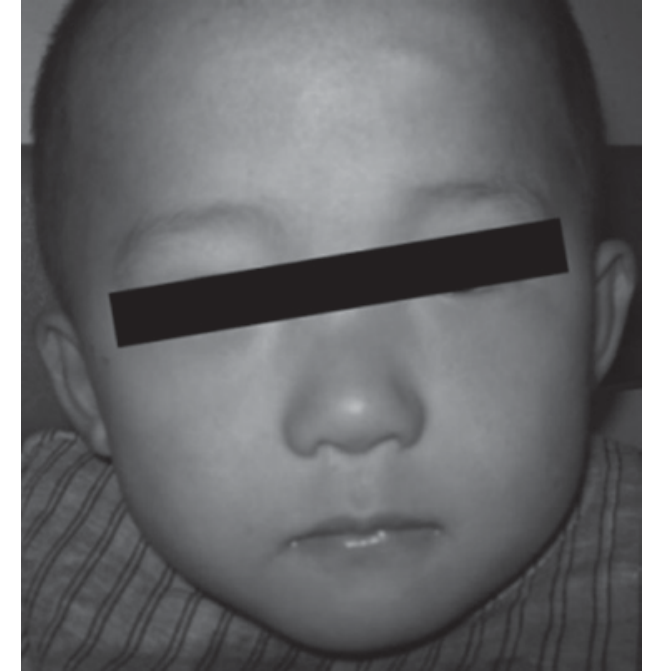

Figure 3. Proband III:7 of the Van der Woude syndrome 2 family.

Consequently, for the diagnosis of VWS, a detailed survey of the patient's family and clinical phenotyping is required.

With the development of molecular biology and the completion of the human genome map, research has made progress regarding the disease genes associated with VWS. In 1954, Van der Woude first described the clinical manifestation of VWS and discussed its hereditary pattern (11). Then, in 1987, Bocian and Walker (12) first reported that these patients were missing chromosome lq32-q41. In 2002, Kondo et al (2) analyzed a large VWS family: No matter what the phenotype of VWS family members, there was an $18 \mathrm{bp}$ fragment missing in the chromosome lq32-q41 areas of the IRF6 genes of the affected individual. Kondo et al (2) successfully cloned the VWS IRF6 disease-causing genes and similar results have also been identified in other VWS families. Therefore, the study demonstrated that VWS had significant phenotypic differences. Certain reports have confirmed that the IRF6 gene is one of the pathogenic genes for VWS $(13,14)$. The present study demonstrated that IRF6 is important in jaw development and fusion. IRF6 gene expression analysis demonstrated that the gene is not only widely expressed in embryonic and adult mice, it is also expressed in adult and fetal tissue. There was a wide range of expression in the secondary cleft palate in 14.5-15 day mouse embryos and adult skin tissue (2).

The IRF6 gene possesses a total length of 2,171 bp, comprising 10 exons. Exon 1,2 and 10 are non-coding regions. The coding regions of IRF6 gene, a total of 1,404 bp, encodes 467 amino acids (2). It is a member of the interferon regulatory factor family and this gene family is characterized by a highly-conserved helix-turn-helix DNA binding domain and a protein binding domain termed Smad-interferon regulatory domain (SMIR) (15). The DNA binding domain mediates the adhesion between IRF6 and the target sequence, while the SMIR domain is necessary to form homologous dimers or heterologous dimers, to translocate dimers to the nucleus, along with other transcription factors in target genes (16). The SMIR domain mediates the morphological development of the mouth-face region through transforming growth factor- $\beta$ signal transduction pathways. At present, the majority of the mutations are in these two domains. According to The 
Table I. Primer sequences and fragment lengths for IRF6 gene in polymerase chain reaction.

\begin{tabular}{llc}
\hline Primer name & \multicolumn{1}{c}{ Primer sequences (5'-3') } & Fragment length (bp) \\
\hline IRF6-3F & CCACCTGGCACAGCTTATTC & 492 \\
IRF6-3R & AGACATGCCCCCAAAAGAG \\
IRF6-4F & TCTCTGTAAATCGGGGTTGG & 527 \\
IRF6-4R & TTTGTTTTCTGGGTCCTTCC & 496 \\
IRF6-5F & GGAGGTCCTTCCATGAGAGA & 500 \\
IRF6-5R & GGGATAACTGAGCCAACAGG \\
IRF6-6F & GGAGCAGGGGAACCTTATTT & \\
IRF6-6R & CCTGGTGACTCATGGGCTA & 700 \\
IRF6-7F & CTATGGCAGTGGCCTTCCT & 430 \\
IRF6-7R & GTTGCCTAGGTCACCTCCAA & \\
IRF6-8F & TGACTAATGTGACCCAGGAACT & 600 \\
IRF6-8R & AGCATGAGTCCTACTGCACAAA & \\
IRF6-9F & TCATTTGAACCAACTTCCACAG & \\
IRF6-9R & AGTCTGAAGGGTGATTTTCCA & \\
\hline
\end{tabular}

F, forward; R, reverse.

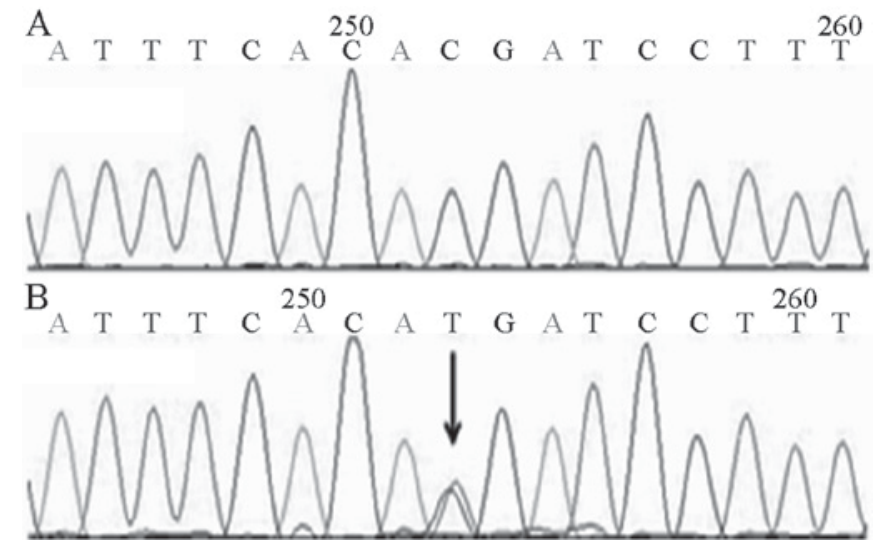

Figure 4. The c.1234C $>\mathrm{T}$ heterozygous mutation of interferon regulatory factor 6 gene. (A) Regular nucleotide sequence, (B) mutated nucleotide sequence. Arrow indicates mutation base.

Human Gene Mutation Database statistics (www.hgmd.cf.ac .uk/ac/index.php), as of September 2012 there are a total of 219 IRF6 gene mutations, including 191 IRF6 gene mutations identified in patients with VWS. These mutations include missense/nonsense, control area, frameshift and various other different forms of mutations, however the majority appear to be missense/nonsense mutations.

The VWS families in the present study exhibited two mutations, which were located on exon 9 in the coding region of the IRF6 gene. In the first VWS family, c.1234C $>$ T resulted in a truncation mutation, and the 412th amino acid appeared as an early termination codon (p.R412X). The first generation of VWS family members with this mutation were generally characterized by concave lower lip or fistula; the second and third generations demonstrated serious cleft palate. In 2009 , this mutation was reported in a European VWS family (17). The emergence of these nonsense mutations resulted in partial

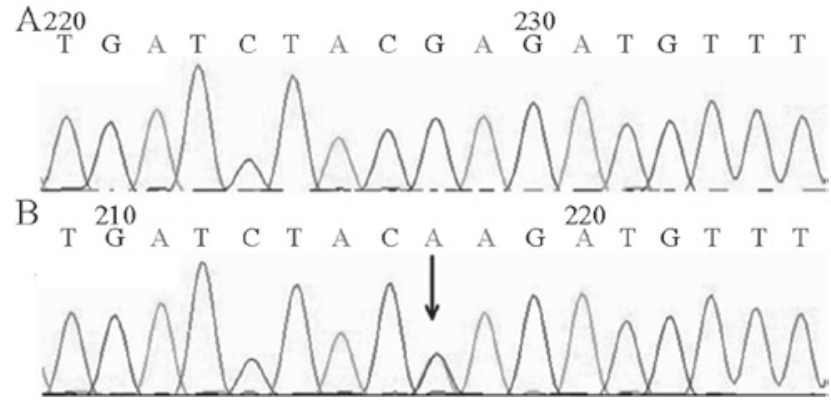

Figure 5. The c.1210G $>$ A heterozygous mutation of interferon regulatory factor 6 gene. (A) Regular nucleotide sequence, (B) mutated nucleotide sequence. Arrow indicates mutation base.

IRF6 gene encoding protein and thus loss of normal function: IRF6 decreased, leading to developmental disorders in the maxillofacial development process that affected the lips and/or jaw. In the present study, the second VWS family carried the c.1210G $>$ A mutation, an alteration in the 404th amino acid which replaced glutamic acid with lysine (p.E404K). The first-generation members of this VWS family did not carry the IRF6 mutations and were not VWS cases; II:3 may therefore be an instance of a novel mutation.

In 2009, de Lima (18) reported this genetic mutation in VWS. However, the pathogenic mechanism of the mutation remains to be elucidated. In the present study, the three websites consulted (www.genetics.bwh.harvard.edu, www. sift.jcvi.org and www.pantherdb.org) all suggested that the mutations may result in damaging effects on the IRF6 protein. It was hypothesized that as the mutation was only 10 amino acids from the downstream SMIR function structure domain, the mutation may lead to an alteration in the nature of the amino acids, thus affecting the function of the protein. Two mutations in line with the White family (17) were detected in the mutation. In order to confirm if these two mutations 
are VWS hot spot mutations, further samples and analyses are required.

The two mutations identified are VWS heterozygous mutations, resulting in an insufficient haploid dose of the IRF6 gene, affecting the maxillofacial growth of the patients. However, it was also identified that even the same mutations present in patients from the same family exhibited different clinical phenotypes. The propositus of the second VWS Family (III:7) was only characterized by simple cleft palate, whereas the other two patients in the family were characterized by lip and cleft palate with fistula phenotype. In the same family, syndrome and non-syndrome occurred in patients with cleft lip and palate cleft lip, which is relatively rare. Therefore, the IRF6 gene sequencing results for the 3 patients of the second VWS family were analyzed. The results demonstrated that II:3 and III:3 exhibited lip fistula and carried SNP loci (rs2235373), and the propositus simply carried p.E404k heterozygous mutations. However, the rs 2235373 polymorphic loci was located in the IRF6 gene downstream of the exon 7 of $135 \mathrm{bp}$, so its effect on IRF6 encoding protein requires further studies. Currently, there are no reports that these mutations affect IRF6 protein production, therefore further studies are required. Salahshourifar (19) summarized the gene mutation and IRF6 introns and exons of mutation loci in different VWS phenotypes. VWS patients with cleft palate II phenotype carried rs2235373 and exon 7 p.V274I loci. These phenomena illustrated that rs2235373 may be associated with the VWS phenotype loci. The present study also identified a phenotypic generational aggravating phenomenon in the first VWS family, a phenomenon that has occurred in other similar studies in the literature (20-22). It has additionally been suggested that there may be other modifiers in virulent VWS genes (23). Due to the diversity of clinical phenotypes in VWS patients, even individuals with a family genetic disposition, may be misdiagnosed by antenatal ultrasound examination; however, genetic testing may help to remedy these shortcomings.

Recently there have been a large number of studies on the VWS IRF6 gene in patients with novel mutations $(17,18,23,24)$. From these studies, it may be suggested that the majority of mutations associated with VWS are point mutations, although splice site mutations resulted in a more intense clinical phenotype $(17,19,25)$. The majority of these reports were from Asian patients and consistent with the previously identified higher percentage incidence of cleft palate among them. Tan et al (25) identified that the 396C-T mutation in the IRF6 gene fragment may be a IRF6 pathogenic factor. In addition, the study suggests that the IRF6 gene mutation distribution in VWS patients is not random and the majority of mutations are located on exons $3,4,7$ and 9 , with $\sim 1 / 5$ of the mutations present on exon $9(18-20,23,24,26)$. The pathogenic mutation research for VWS has been performed with a relatively small Chinese cohort. The present study collected data from two VWS families and identified that the pathogenic mutations were all located on exon 9. Therefore, the exon 9 may be a hot spot mutation in VWS occurring in Chinese patients. It will be necessary to expand the sample size in the future in order to conduct in-depth research.

In conclusion, the accumulation of these clinical data and the study of pathogenic mutations may prove useful for the differential diagnosis of VWS and NSCL/P. In addition, the results of the present study may identify potential therapeutic targets. They also provide the basis and support for genetic counseling and prenatal diagnosis, and the prevention of cleft lip and palate with gene therapy. With the advent of the genome, improved pathogenic mutation research into the syndrome of cleft lip and palate may be achieved, and the association between VWS and NSCL/P analyzed and clarified in the Chinese population. It is hypothesized that genetic research regarding NSCL/P and study of the underlying molecular mechanism of the occurrence of cleft lip and palate, will make greater progress in the future. The authors believe that the study of mutations in this syndrome will aid therapeutic research and improve the quality of life in patients with VWS.

\section{Acknowledgements}

The present study was funded by the Science and Technology Project of Hebei Province (grant no. 1427781D) and the Science and Technology Research and Development Program of Handan (grant no. 1223108085).

\section{References}

1. Schutte BC, Bjork BC, Coppage KB, Malik MI, Gregory SG, Scott DJ, Brentzell LM, Watanabe Y, Dixon MJ and Murray JC: A preliminary gene map for the Van der Woude syndrome critical region derived from $900 \mathrm{~kb}$ of genomic sequence at 1q32-q41. Genome Res 10: 81-94, 2000.

2. Kondo S, Schutte BC, Richardson RJ, Bjork BC, Knight AS, Watanabe Y, Howard E, de Lima RL, Daack-Hirsch S, Sander A, et al: Mutations in IRF6 cause Van der Woude and popliteal pterygium syndromes. Nat Genet 32: 285-289, 2002.

3. Burdick AB: Genetic epidemiology and control of genetic expression in van der Woude syndrome. J Craniofac Genet Dev Biol Suppl 2: 99-105, 1986.

4. Pingul MM and Quintos JB: Van der woude syndrome (lip pit-cleft lip syndrome). J Pediatr 164: 1235, 2014.

5. Peyrard-Janvid M, Pegelow M, Koillinen H, Larsson C Fransson I, Rautio J, Hukki J, Larson O, Karsten AL and Kere J: Novel and de novo mutations of the IRF6 gene detected in patients with Van der Woude or popliteal pterygium syndrome. Eur J Hum Genet 13: 1261-1267, 2005.

6. Mijiti A,Ling W, Guli and Moming A: Association of single-nucleotide polymorphisms in the IRF6 gene with non-syndromic cleft lip with or without cleft palatein the Xinjiang Uyghur population. Br J Oral Maxillofac Surg 53: 268-274, 2015.

7. XQ Ye, B Zhou and H Jin: Clinical and genetic features in five chinese kindreds with Van der Woude syndrome. Med J Wuhan University 26: 609-611, 2005

8. Burdick AB, Bixler D and Puckett CL: Genetic analysis in families with van der Woude syndrome. J Craniofac Genet Dev Biol 5: 181-208, 1985 .

9. Hersh JH and Verdi GD: Natal teeth in monozygotic twins with Van der Woude syndrome. Cleft Palate Craniofac J 29: 279-281, 1992.

10. Dixon MJ, Marazita ML, Beaty TH and Murray JC: Cleft lip and palate: Understanding genetic and environmental influences. Nat Rev Genet 12: 167-178, 2011.

11. Van Der Woude A: Fistula labii inferioris congenita and its association with cleft lip and palate. Am J Hum Genet 6: 244-256, 1954.

12. Bocian M and Walker AP: Lip pits and deletion 1q32-41. Am J Med Genet 26: 437-443, 1987.

13. Zhou Q, Li M, Zhu W, Guo J, Wang Y, Li Y and Li S: Association between interferon regulatory factor 6 gene polymorphisms and nonsyndromic cleft lip with or without cleft palate in a Chinese population. Cleft Palate Craniofac J 50: 570-576, 2013.

14. Leslie EJ, Standley J, Compton J, Bale S, Schutte BC and Murray JC: Comparative analysis of IRF6 variants in families with Van der Woude syndrome and popliteal pterygium syndrome using public whole-exome databases. Genet Med 15: 338-344, 2013. 
15. Eroshkin A and Mushegian A: Conserved transactivation domain shared by interferon regulatory factors and Smad morphogens. J Mol Med (Berl) 77: 403-405, 1999.

16. Mamane Y, Heylbroeck C, Génin P, Algarté M, Servant MJ, LePage C, DeLuca C, Kwon H, Lin R and Hiscott J: Interferon regulatory factors: The next generation. Gene 237: $1-14,1999$.

17. Houweling AC, Gille JJ, Baart JA, van Hagen JM and Lachmeijer AM: Variable phenotypic manifestation of IRF6 mutations in the Van der Woude syndrome and popliteal pterygium syndrome: Implications for genetic counseling. Clin Dysmorphol 18: 225-227, 2009.

18. de Lima RL, Hoper SA, Ghassibe M, Cooper ME, Rorick NK Kondo S, Katz L, Marazita ML, Compton J, Bale S, et al: Prevalence and nonrandom distribution of exonic mutations in interferon regulatory factor 6 in 307 families with Van der Woude syndrome and 37 families with popliteal pterygium syndrome. Genet Med 11: 241-247, 2009.

19. Salahshourifar I, Wan Sulaiman WA, Halim AS and Zilfalil BA: Mutation screening of IRF6 among families with non-syndromic oral clefts and identification of two novel variants: Review of the literature. Eur J Med Genet 55: 389-393, 2012.

20. Soekarman D, Cobben JM, Vogels A, Spauwen PH and Fryns JP: Variable expression of the popliteal pterygium syndrome in two 3-generation families. Clin Genet 47: 169-174, 1995.
21. Marazita ML, Lidral AC, Murray JC, Field LL, Maher BS, Goldstein McHenry T, Cooper ME, Govil M, Daack-Hirsch S, Riley B, et al: Genome scan, fine-maping, and candidate gene analysis of non-syndomic cleft lip with or without cleft palate reveals phenotype-sppecific diferences in linkage and association results. Hum Hered 68: 151-170, 2009.

22. Jugessur A, Shi M, Gjessing HK, Lie RT, Wilcox AJ, Weinberg CR, Christensen K, Boyles AL, Daack-Hirsch S, Nguyen TT, et al: Fetal genetic risk of isolated cleft lip only (CLO) versus isolated cleft lip and palate (CLP): A sub-phenotype analysis usingtwo population-based studies of orofacial clefts in Scandinavia. Birth Defects Res A Clin Mol Teratol 91: 85-92, 2011.

23. de la Garza G, Schleiffarth JR, Dunnwald M, Mankad A, Weirather JL, Bonde G, Butcher S, Mansour TA, Kousa YA, Fukazawa $\mathrm{CF}$, et al: Interferon regulatory factor6 promotes differentiation of the peridermby activating expression of Grainyhead-like 3. J Invest Dermatol 133: 68-77, 2013.

24. Peyrard-Janvid M, Leslie EJ, Kousa YA, Smith TL, Dunnwald M, Magnusson M, Lentz BA, Unneberg P, Fransson I, Koillinen HK, et al: Dominant mutations in GRHL3 cause Van der Woude Syndrome and disrupt oral periderm development. Am J Hum Genet 94: 23-32, 2014.

25. Tan EC, Lim EC, Yap SH, Lee ST, Cheng J, Por YC and Yeow V: Identification of IRF6 gene variants in three families with Van der Woude syndrome. Int J Mol Med 21: 747-751, 2008.

26. Morton NE: Sequential tests for the detection of linkage. Am J Hum Genet 7: 277-318, 1955. 